\title{
Comparative Study of Ultrasonography Techniques in Predicting Histopathological Lesion of Canine Tumours
}

\author{
Sagar Sahoo ${ }^{1}$, Biswadeep Jena ${ }^{1 *}$, Indramani Nath ${ }^{1}$, Jayakrushna Das ${ }^{1}$, Sidhartha Sankar Behera ${ }^{1}$, \\ Prasana Kumar Rath ${ }^{2}$, Shuvranshu Sekhar Biswal ${ }^{3}$ and Kautuk Kumar Sardar ${ }^{4}$ \\ ${ }^{1}$ Department of Veterinary Surgery and Radiology, College of Veterinary Science and Animal Husbandry, Odisha University of \\ Agriculture and Technology, Bhubaneswar, Odisha, INDIA \\ ${ }^{2}$ Department of Veterinary Pathology, College of Veterinary Science and Animal Husbandry, Odisha University of Agriculture \\ and Technology, Bhubaneswar, Odisha, INDIA \\ ${ }^{3}$ Department of Veterinary Gynaecology and Obstetrics, College of Veterinary Science and Animal Husbandry, Odisha University \\ of Agriculture and Technology, Bhubaneswar, Odisha, INDIA \\ ${ }^{4}$ Department of Veterinary Pharmacology and Toxicology, College of Veterinary Science and Animal Husbandry, Odisha \\ University of Agriculture and Technology, Bhubaneswar, Odisha, INDIA \\ *Corresponding author: B Jena; E-mail: biswadeep44@gmail.com
}

Received: 13 June, 2020

Revised: 13 July, 2020

Accepted: 26 July, 2020

\begin{abstract}
Present study was to evaluate and standardize different ultrasonography techniques to predict malignancy of canine tumours. The study was conducted on 28 canine patients (20 females and 8 males) with visible tumour masses with age ranging from 5 to 13 years of age and body weight from 11 to $37 \mathrm{Kg}$, for a period of 8 months. The ultrasonographic variables for different techniques (B-mode, Colour Doppler and stress elastography) were measured and recorded. The tumour masses after excision were submitted for routine histopathological examination. Statistical analysis was performed using analysis of variance and paired " $\mathrm{t}$ " test using IBM SPSS statistical packages. The B-mode and Colour Doppler ultrasonography had a low to moderate sensitivity, specificity and accuracy in predicting the malignancy of canine tumours. The stress elastography was a reliable and non-invasive technique to predict the malignancy in case of canine tumours with highest level of sensitivity, specificity and accuracy.
\end{abstract}

\section{HIGHLIGHTS}

( Stress elastography predicted malignant canine tumour efficiently.

O Shear wave velocity, beyond $3.35 \mathrm{~m} / \mathrm{sec}$, predicted malignancy with highest accuracy.

( $\mathrm{B}$-mode and Colour Doppler predicted malignant canine tumour inaccurately.

Keywords: B-Mode, Canine, Colour Doppler, Elastography, Tumour

A study that was conducted on a series of more than 2000 necropsy cases to determine the cause of death suggested that cancer is a measure cause of pet animal death. This study also revealed that $45 \%$ of dogs that lived up to 10 years or older died of cancer. A Mark Morris Foundation Animal health survey done in 2005 ranked cancer as the largest concern (41\%) among pet owners. Regardless of the exact numbers, one of the leading killer diseases among pet animals is cancer and is becoming a burgeoning concern for pet owners as it relates to the health status of their companions (Carvalho et al., 2015).

Diagnosis and treatment are the two sides of same coin. This becomes immensely important for the pets with

How to cite this article: Sahoo, S., Jena, B., Nath, I., Das, J., Behera, S.S., Rath, P.K., Biswal, S.S. and Sardar, K.K. (2020). Comparative study of ultrasonography techniques in predicting histopathological lesion of canine tumours. J. Anim. Res., 10(4): 641-649.

Source of Support: None; Conflict of Interest: None क क 
cancer. In veterinary oncology, there are some established cancer treatments available such as chemotherapy, immunotherapy, radiation therapy, molecular therapy and surgery etc., but the diagnostic techniques for the cancer detection are still underdeveloped. In this context, histopathology stands as the lone warrior to diagnose the cancer. It is the gold standard diagnostic modality for typing, grading and staging of cancer. But this process takes minimum 7-10 days to get the result. As a single day in a cancer patient's life is precious, the need for faster and better diagnostic imaging is emerging. An early and sensitive diagnosis represents a better prognosis. The sophistication of advanced imaging modalities is continuing to increase exponentially. Each modality has certain advantages and disadvantages depending on the cost, availability, sensitivity and specificity. So advanced imaging techniques play an important role in the tailoring of cancer therapy in veterinary patients.

Ultrasound imaging has the ability to evaluate the internal structure with moderate cost, higher degree of sensitivity and moderate degree of specificity. In human medicine, ultrasound imaging has replaced the survey radiograph in the diagnosis of cancer, up to an extent (Dixon et al., 1992). But in veterinary practice, ultrasound imaging is still being used as a guide to take fine needle aspirational biopsies only. But with the advent of modern ultrasound techniques like B-Mode ultrasonography, Colour Doppler ultrasonography and stress elastography etc.; it is possible to study multiple quantitative and qualitative parameters of tumours to differentiate benign versus malignant neoplasm in various organs like mammary glands, thyroid, prostate, kidney and lymph nodes (Forrest et al., 2007).

Review of literature suggests that research data regarding modern ultrasound imaging techniques like B-Mode ultrasonography, Colour Doppler ultrasonography and stress elastography etc., are lacking and no study has attempted to correlate the aforementioned ultrasound imaging techniques and the histopathological typing of canine tumours.

Therefore, the present study was planned to evaluate vis-à-vis compare the diagnostic accuracy of B-Mode ultrasonography, Colour Doppler ultrasonography and stress elastography in predicting types of canine neoplasm prior to their routine histopathological examinations as a reference and to standardise the values of different qualitative and quantitative variables of B-Mode ultrasonography, Colour Doppler ultrasonography and stress elastography in predicting types of neoplasm in canines.

\section{MATERIALS AND METHODS}

Pet dogs (Canis lupus familiaris) of either sex and of any age, presented with visible tumorous mass were made the subject of study. History of animal regarding breed, age, sex, body weight, general complaints of the condition that necessitate medical and/or surgical interventions, time of occurrence and any prior treatment given was recorded.

\section{Details of ultrasound machine and its probes}

Ultrasonography was performed with Make - Wipro GE, Model - Logiq F8 Expert ultrasound machine (Solingen, Germany). 8C micro convex multifrequency probe (frequency range $3.0-10.0 \mathrm{MHz}$ ) was used for grey scale 2D (B-Mode) imaging and Colour Doppler ultrasonography. L6-12 linear multifrequency probe (frequency range $6.0-12.0 \mathrm{MHz}$ ) was used for stress elastography.

\section{Qualitative parameters observed}

Qualitative parameters of visible tumour mass analysed by the B - Mode ultrasonography were echo-texture (homogenous or heterogeneous), contours/margins (defined or undefined) and invasiveness (present or absent).

Qualitative parameters analysed by Spectral Doppler ultrasonography were vascular visualization by colour flow (present or absent), localization (peripheral, central or diffuse), vessel type (perinodular, mosaic or network), characteristics (arterial or turbulent), patterns (high resistivity, intermediate or low).

Qualitative parameter analysed by stress elastography was deformability (deformable or non-deformable).

\section{Quantitative parameters observed}

Quantitative parameters of visible tumour mass analysed by B - Mode ultrasonography were longitudinal width $(\mathrm{cm})$, longitudinal length $(\mathrm{cm})$, width: length ratio; 
transverse width $(\mathrm{cm})$, transverse height $(\mathrm{cm})$, width: height ratio.

Quantitative parameters analysed by Spectral Doppler ultrasonography were systolic velocity $(\mathrm{Vs})(\mathrm{cm} / \mathrm{s})$, diastolic velocity $(\mathrm{Vd})(\mathrm{cm} / \mathrm{s})$, resistive index $(\mathrm{RI})$ and pulsatility index (PI).

Quantitative parameter analysed by stress elastography was shear wave velocity (SWV) $(\mathrm{m} / \mathrm{s})$.

\section{Histopathological observation}

The tumour mass was excised under routine general anaesthetic protocol. 2 to 4 numbers of $1 \times 1 \times 1 \mathrm{~cm}^{3}$ sample specimens from areas representing different consistencies or colours were collected and subjected to routine histopathological examination.

\section{STATISTICAL ANALYSIS}

The quantitative data were presented as Mean \pm Standard Error (SE) and qualitative data as percentage. Qualitative ultrasound variables were compared between benign and malignant masses by paired ' $\mathrm{T}$ ' test using IBM SPSS version - 20. For ultrasonography parameters that showed significance, the cut-off point, sensitivity, specificity, accuracy, and area under curve (AUC) were calculated using histopathological classification as a reference for receiver-operating characteristic (ROC) curve analysis in a logistic regression model aimed at assessing and comparing the diagnostic performance of each technique. For hypothesis testing $\mathrm{P}<0.01$ and $\mathrm{P}<0.05$ were considered and the level of significance was noted (Snedecor and Cochran, 2004).

\section{RESULTS AND DISCUSSION}

Different qualitative and quantitative variables of ultrasonographic techniques viz. B-Mode ultrasonography, Colour Doppler ultrasonography and Stress elastography were correlated with histopathological findings to establish a non-invasive ultrasonographic standard to predict the type of canine tumour mass. This will expedite the subsequent therapeutic vis-à-vis surgical interventions to prolong the well-being of canine cancer patients. Our study didn't aim to replace the histopathological evaluation of tumour as it still remains the gold standard. The main aim of our study was to give a head start of 07 to 10 days, which was the normal duration for histopathological examination, for our therapeutic vis-à-vis surgical interventions for treatment of canine cancer patients.

\section{Histopathological examination-based observations}

The present study was conducted on 28 pet dogs with visible tumorous mass. In toto, 28 samples suspected for neoplastic mass were included in the present research programme comprising of mammary tumour $(n=17)$, lipoma $(n=2)$, cutaneous tumour $(n=7)$ and vaginal polyp $(n=2)$. Present study concluded highest incidence of mammary tumour $(60.71 \%)$ which corresponded to Patel et al. (2019). Mammary tumours in canines as observed during the study mostly prevalent with inguinal pair of teats, predominantly 9-13 years age groups and in Labrador breeds which is in accordance with Vascellari et al. (2009) and Cassali et al. (2012). Out of 28 tumour masses evaluated, 11 (39.3\%) were histopathologically classified as malignant and $17(60.7 \%)$ as benign masses by a single experienced pathologist, which was in concomitant with findings of Nair et al. (2007).

\section{Qualitative parameters observed under B - Mode ultrasonography}

Heterogenous echotexture, defined contours/margins and absence of invasiveness were predominantly observed in both benign and malignant type tumours (Table 1).

Table 1: Qualitative parameters of canine neoplasms under B Mode Ultrasonography

\begin{tabular}{lllll}
\hline Variables & Parameters & Benign & Malignant & P-value \\
\hline Echotexture & Homogenous (\%) & 17.65 & 0.00 & \multirow{2}{*}{0.151} \\
& Heterogenous (\%) & 82.35 & 100.00 & \\
Contours/ & Defined (\%) & 100.00 & 90.90 & 0.220 \\
Margins & Undefined (\%) & 0.00 & 9.10 & \\
Invasiveness & Present (\%) & 0.00 & 9.10 & 0.220 \\
& Absent (\%) & 100.00 & 90.90 & \\
\hline
\end{tabular}

Echotexture, margin, and invasiveness evaluation by B-mode ultrasonography did not allow differentiation of canine tumours, probably due to the high variability in the morpho-structural characteristics of each tumour type and consequently limiting the applicability of this method, in 
agreement with studies in women with breast carcinomas (Feliciano et al., 2012a; Feliciano et al., 2012b; Feliciano et al., 2017; Feliciano et al., 2018) (Fig. 1).
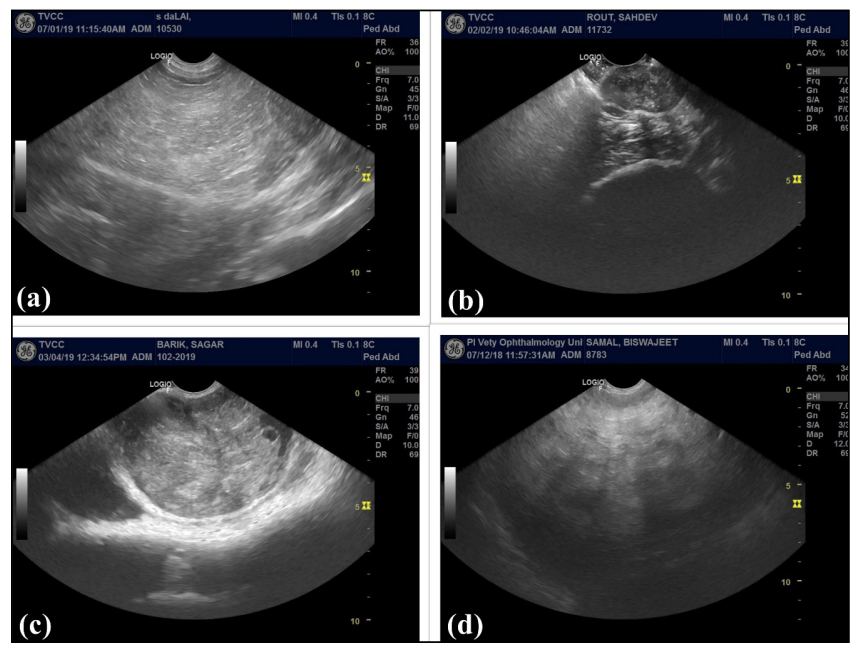

Fig. 1: Qualitative parameters of canine neoplasms under B Mode Ultrasonography. Showing (a) homogenous echotexture of a benign canine tumour mass; (b) heterogenous echotexture of a malignant canine tumour mass; (c) defined contour/ margin of a benign canine tumour mass; (d) undefined contour/ margin of a malignant canine tumour mass

There was a difference in echogenicity between the different groups of tumours. It has previously been reported that all the malignant canine mammary tumours had a varied echo texture, although, only 11 malignant mammary tumours were evaluated (de Bulnes et al., 1998). Results of our study have confirmed the heterogenic nature of tumours in dogs. The ultrasonographic finding of varied echogenicity correlated with tissue heterogeneity detected histologically. The varied echogenicity observed particularly in tumours may be a consequence of the development of necrosis, cysts, oedema, haemorrhage, or increased interstitial pressure or cartilage and mineralization within the tumour (Nyman et al., 2006a; Nyman et al., 2006b).

In present ultrasonographic images, the tumours were rarely seen invading tissue layers beneath their place of occurrence; although invasiveness was more commonly observed with malignant tumours than benign tumours (Nyman et al., 2006a). This lack of correlation is surprising, as a distinct difference between the tumour groups was expected. It can be argued that the reason for this may be that we should have used another scanning definition of invasion or that reactive, hyperplastic, or inflammatory tissue surrounding some tumours may affect delineation of the tumour borders.

\section{Quantitative parameters observed under B - Mode ultrasonography}

All the quantitative measurement parameters observed under B - Mode ultrasonography had shown significant $(\mathrm{P}<0.05$ and $\mathrm{P}<0.01)$ difference between malignant and benign type of tumours (Table 2 ).

Table 2: Quantitative parameters of canine neoplasms under B - Mode ultrasonography

\begin{tabular}{llll}
\hline Parameters & Benign & Malignant & P-value \\
\hline $\begin{array}{l}\text { Longitudinal Length } \\
(\mathrm{cm})\end{array}$ & $10.88 \pm 2.45$ & $9.98 \pm 1.21^{* *}$ & 0.000 \\
Longitudinal Width $(\mathrm{cm})$ & $7.81 \pm 1.57$ & $6.94 \pm 0.74^{* *}$ & 0.002 \\
Length/Width Ratio & $1.49 \pm 0.11$ & $1.50 \pm 0.13^{*}$ & 0.042 \\
Transverse Length $(\mathrm{cm})$ & $11.56 \pm 2.79$ & $10.25 \pm 1.45^{* *}$ & 0.000 \\
Transverse Height $(\mathrm{cm})$ & $5.54 \pm 0.93$ & $6.27 \pm 0.57^{*}$ & 0.020 \\
Length/Height Ratio & $2.08 \pm 0.17$ & $1.71 \pm 0.27 * *$ & 0.001 \\
\hline
\end{tabular}

*Significantly different from the base value $(\mathrm{P}<0.05)$; **Significantly different from the base value $(\mathrm{P}<0.01)$.

The large size of mammary masses has been described as a poor prognosis factor in female dogs, especially when associated with regional abnormalities of the lymphoid tissue, rapid growth, and/or ulceration (Feliciano et al., 2018). In literature, the use of B-mode ultrasonography to analyse 30 malignant, eight atypical, and 11 benign mammary lesions in female dogs concluded that the larger masses were correlated to high-grade malignancy and worse prognosis (Nyman et al., 2006a).

However, in the present study, only length: width ratio greater than 1.49 obtained by B-mode ultrasonography enabled the identification of special type carcinomas with acceptable accuracy. These findings are in agreement with previous reports, which have identified this ratio to be an indicator of malignancy in mammary tumors in women and female dogs (Feliciano et al., 2017). Thus, this parameter can be used as a simple and economical tool in the diagnosis of mammary neoplasms in female dogs.

Predictive performance variables of B -Mode ultrasonography revealed low sensitivity (54.54\%) 
and moderate specificity (70.58\%) as well as accuracy (64.28\%). Hence, our study was in disagreement with some reports that had considered variables of B -Mode ultrasonography to be indicative of malignant tumours (Nyman et al., 2006b; Soler et al., 2016). Corroborating the results from this study, B-mode ultrasonography has been considered to be a technique with low specificity when used as an isolated method of evaluation (Gokhale et al., 2009).

\section{Qualitative parameters observed under Colour Doppler ultrasonography}

Table 3: Qualitative parameters of canine neoplasms under Colour Doppler ultrasonography

\begin{tabular}{lllll}
\hline Variables & Parameters & Benign & Malignant & $\begin{array}{l}\text { P- } \\
\text { value }\end{array}$ \\
\hline Vascularisation & Present (\%) & 100.00 & 100.00 & - \\
& Absent (\%) & 0.00 & 0.00 & \\
Localisation & Peripheral (\%) & 70.60 & 36.36 & 0.031 \\
& Central (\%) & 29.40 & 45.46 & \\
& Diffuse (\%) & 0.00 & 18.18 & \\
Vessel Type & Perinodular (\%) & 82.35 & 45.46 & 0.041 \\
& Mosaic (\%) & 11.76 & 27.27 & \\
& Network (\%) & 5.89 & 27.27 & \\
Characteristics & Arterial (\%) & 88.24 & 45.46 & 0.013 \\
& Turbulent (\%) & 11.76 & 54.54 & \\
Patterns & High Resistivity & 52.94 & 27.27 & 0.864 \\
& (\%) & & & \\
& Intermediate (\%) & 17.64 & 63.63 & \\
& Low Resistivity & 29.42 & 9.10 & \\
& (\%) & & & \\
\hline
\end{tabular}

Vascularisation was present in all the cases. Peripheral localisation of blood vessels was significantly $(\mathrm{P}<0.05)$ predominant in benign tumour masses, whereas central localisation of blood vessels was significantly $(\mathrm{P}<0.05)$ predominant in malignant tumour masses. Peri-nodular blood vessels were significantly $(\mathrm{P}<0.05)$ predominant in both benign and malignant tumour masses. Arterial blood flow was significantly $(\mathrm{P}<0.05)$ predominant in benign tumour masses, whereas turbulent blood flow was significantly $(\mathrm{P}<0.05)$ predominant in malignant tumour masses. Though non-significant $(\mathrm{P}>0.05)$, benign tumour masses had shown high resistive pattern of blood flow, whereas the malignant tumour masses had shown intermediate pattern blood flow (Table 3 and Fig. 2).
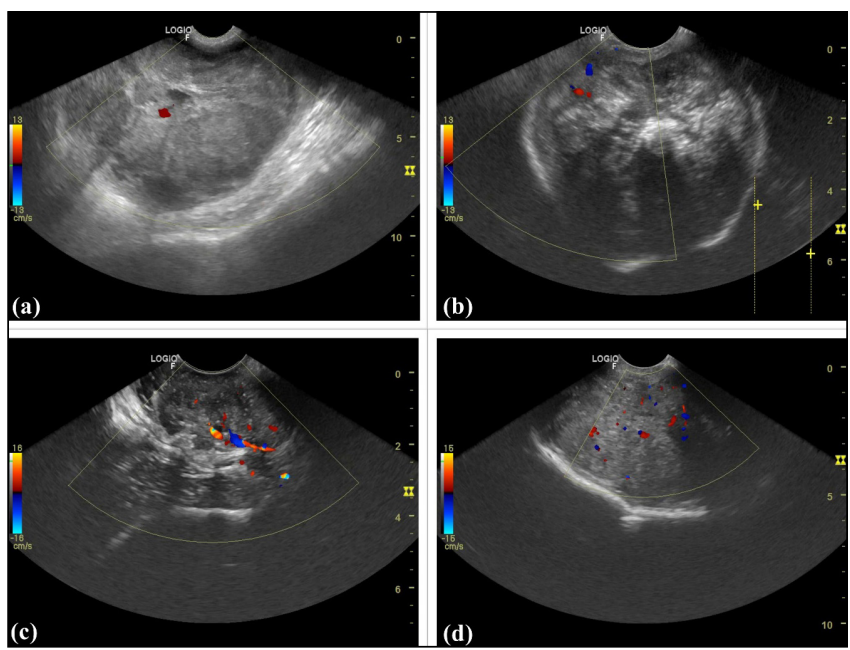

Fig. 2: Qualitative parameters of canine neoplasms under Colour Doppler ultrasonography. Showing (a) central localisation, perinodular vessels and arterial blood flow; (b) peripheral localisation, perinodular vessels and arterial blood flow; (c) peripheral localisation, mosaic vessels and turbulent blood flow; (d) network localisation, perinodular vessels and arterial blood flow

Colour Doppler ultrasonography can be used to detect angiogenesis within solid tumours, and several authors have suggested the possibility for differentiating malignant and benign tumours. It has been reported in some studies that vessels in malignant tumours divide more than in benign (Stuhrmann et al., 2000). But our study failed to differentiate between benign and malignant tumours with regard to the number of vessels to the tumour.

In this study the vascular localisation within the tumour was subjectively divided into three groups: peripheral, central and diffuse. As per reports, benign tumours primarily have a larger blood vessel in the periphery of the tumour, whereas malignant tumours were expected to have an increased number of smaller vessels throughout the tumour as a result of more forceful angiogenesis, as particularly malignant tumour induces aberrant feeding vessels (Nyman et al., 2006b). Similar observations were found in our study.

In this study the vascular type within the tumour was subjectively divided into three groups: perinodular, mosaic and network. Malignant tumours will most likely have 
a high micro-vessel density as a result of an angiogenic response, particularly in certain areas of the tumour. Benign tumours are not expected to have as high as micro-vessel density as malignant tumours because they are usually growing more slowly. Nevertheless, they may contain larger, more-developed vessels; ultrasonographically, this would appear as a large total flow area (Nyman et al., 2006a). Results of other studies have suggested that bifurcations and trifurcations of vessels may be an indication of malignancy (Ohlerth and Kaser-Hotz, 2003) which resulted in higher \% of mosaic and network type vessels as observed in our study.

The results of our study indicate that intra-tumoral blood flow can be detected and evaluated via Colour Doppler ultrasonography and the use of Doppler ultrasonography offers a non-invasive method of assessing characteristics of vascularization in naturally occurring tumours in dogs. In most of the benign tumour masses, the vessels had unidirectional arterial flow which was governed by either blue or red colour coding under Colour Doppler ultrasonography. On the other hand, most of the malignant tumours had turbulent blood flow which was supported by yellow or greenish colour coding under Colour Doppler ultrasonography due to randomization of blood flow direction.

In this study the vascular pattern within the tumour was subjectively divided into three groups: high resistivity, intermediate and low resistivity. Most of the benign tumour masses showed high resistivity pattern of blood flow attributed to perinodular vessels and arterial blood flow. On the contrary, the malignant tumour masses showed an intermediate pattern of blood flow due to increased micro vessel density and turbulent pattern of blood flow. With a sensitivity of $63.63 \%$, specificity of $70.58 \%$, accuracy of $67.85 \%$ and AUC of $67.1 \%$; Colour Doppler qualitative variables cannot be used as a single diagnostic modality to differentiate canine neoplasia.

\section{Quantitative parameters observed under colour doppler ultrasonography}

Though non-significant $(\mathrm{P}>0.05)$, higher mean systolic velocity had been observed in malignant tumour masses. Significantly $(\mathrm{P}<0.05)$ higher mean diastolic velocity had been observed in malignant tumour masses. Nonsignificantly $(\mathrm{P}>0.05)$ higher $\mathrm{RI}$ but significantly $(\mathrm{P}<0.05)$ higher PI were observed in malignant tumour masses (Table 4 and Fig. 3).

Table 4: Quantitative parameters of canine neoplasms under Colour Doppler ultrasonography

\begin{tabular}{llll}
\hline Parameters & Benign & Malignant & P-value \\
\hline Systolic Velocity $(\mathrm{cm} / \mathrm{s})$ & $13.15 \pm 1.35$ & $20.06 \pm 3.72$ & 0.308 \\
Diastolic Velocity $(\mathrm{cm} / \mathrm{s})$ & $7.11 \pm 1.73$ & $10.38 \pm 1.84 *$ & 0.021 \\
Resistive Index (RI) & $0.45 \pm 0.08$ & $0.54 \pm 0.09$ & 0.685 \\
Pulsatility Index (PI) & $0.64 \pm 0.13$ & $1.38 \pm 0.31 *$ & 0.080 \\
\hline
\end{tabular}

*Significantly different from the base value $(\mathrm{P}<0.05)$.
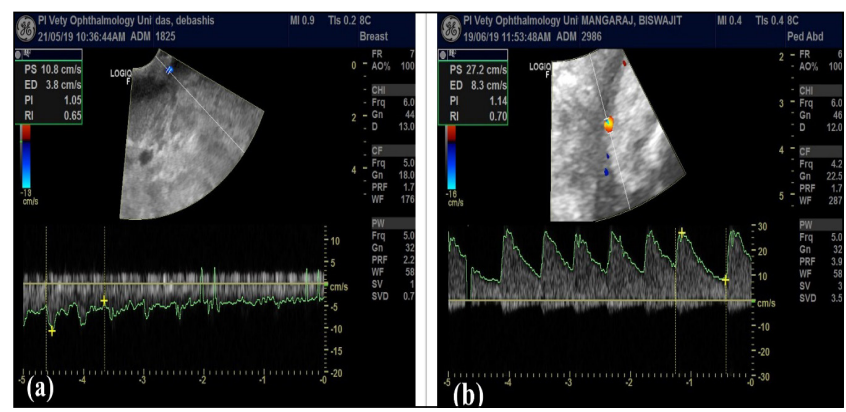

Fig. 3: Quantitative parameters of canine neoplasms under Colour Doppler ultrasonography. Showing vascular indices of (a) benign; (b) malignant tumour masses

Spectral Doppler (vascular index and tracing characteristics) have been shown to be important ultrasonography techniques in the differentiation of malignant and benign tumours (Feliciano et al., 2012a; Feliciano et al., 2012b; Davoudi et al., 2014). High values of $\mathrm{Vs}$ and $\mathrm{Vd}$ observed in the present study have been described as satisfactory indicators of malignancy in mammary masses in humans and animals (Feliciano et al., 2012b). Furthermore, the findings from this study on high resistivity patterns are suggestive of malignancy, as previously described, and may be correlated to the presence of tortuous vascular networks in malignant tumours and demonstrate the influence of neoplastic vascular organization (neovascularization characteristics) in tumoral nutrition (Nyman et al., 2006a; Feliciano et al., 2012b; Davoudi et al., 2014).

With a sensitivity of $45.45 \%$, specificity of $76.47 \%$, accuracy of $64.28 \%$ and AUC of $61.0 \%$; Colour Doppler Quantitative variables cannot be used as a single diagnostic modality to differentiate canine neoplasia. 


\section{Qualitative parameters observed under stress elastography}

Benign tumour masses were found to be significantly $(\mathrm{P}<0.05)$ more deformable, whereas malignant tumour masses were found to be significantly $(\mathrm{P}<0.05)$ more nondeformable (Table 5 and Fig. 4).

Table 5: Qualitative parameters of canine neoplasms under stress elastography

\begin{tabular}{llll}
\hline Parameter & Benign & Malignant & P - value \\
\hline Deformable (\%) & 70.59 & $27.27^{*}$ & 0.024 \\
Not Deformable (\%) & 29.41 & $72.73^{*}$ & \\
\hline
\end{tabular}

*Significantly different from the base value $(\mathrm{P}<0.05)$.

The qualitative stress elastography evaluation in this study revealed that $72.73 \%$ of malignant tumour masses had nondeformable characteristics, in agreement with previous studies that have considered this as malignancy factor (Feliciano et al., 2017; Feliciano et al., 2018). The greater stiffness observed in malignant tumours is a consequence of the stromal reaction induced by the carcinoma, which was associated with increased levels of collagen (Feliciano et al., 2017). Although, stress elastogram had a sensitivity of $81.81 \%$, specificity of $88.23 \%$, accuracy of $85.71 \%$ and AUC of $85.0 \%$; it cannot be used as stand-alone diagnostic modality to differentiate canine neoplasia.

\section{Quantitative parameters observed under stress elastography}

Shear Wave Velocity (SWV) was found to be significantly $(\mathrm{P}<0.05)$ higher in malignant tumour masses with respect to that of benign masses (Table 6 and Fig. 4).

Table 6: Quantitative parameters of canine neoplasms under stress elastography

\begin{tabular}{llll}
\hline Parameters & Benign & Malignant & P-value \\
\hline Shear Wave Velocity $(\mathrm{m} / \mathrm{s})$ & $2.17 \pm 0.22$ & $4.75 \pm 0.30^{*}$ & 0.029 \\
\hline
\end{tabular}

*Significantly different from the base value $(\mathrm{P}<0.05)$.

Using quantitative elastography, the mean shear wave velocity $(\mathrm{m} / \mathrm{sec})$ values of malignant tumours significantly higher with respect to benign tumours $(\mathrm{P}<0.005)$ correlated with low elasticity, non-deformability and rigid consistency (Feliciano et al., 2017; Diaz et al., 2018). Cutoff values above $3.35 \mathrm{~m} / \mathrm{s}$ showed an impressive sensitivity of $90.90 \%$, specificity of $94.11 \%$, diagnostic accuracy of $92.85 \%$ and AUC of $92.5 \%$.
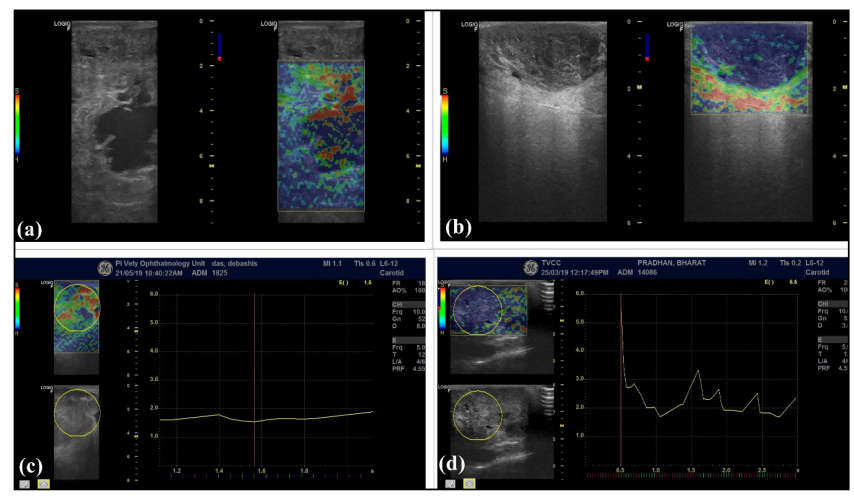

Fig. 4: Quantitative parameters of canine neoplasms under stress elastography. Showing stress elastograms of (a) benign; (b) malignant tumour masses and shear wave velocities of (c) benign; (d) malignant tumour masses. Blue colour denoted hardness, red colour denoted softness and green colour denoted intermediate stiffness of canine tumour masses

The cut-off values in this study were higher than those from previous reports. In human medicine, some authors have reported cut-off values ranging from $2.9-6.4 \mathrm{~m} / \mathrm{s}$, which have been associated to $76-91 \%$ sensitivity and $80-95 \%$ specificity, close values to the observed in the present study and which corroborate the effectiveness of the Stress technique applied in evaluation of tumours in canines. The elastogram characteristics obtained in this study were adequate in the diagnosis of malignancy and similar to those previously described in benign mammary lesions in women and bitches (Feliciano et al., 2017; Feliciano et al., 2018).

The current study has some limitations that must be addressed. Although the sample size was adequate $(\mathrm{n}=$ 28 ), the prevalence of malignant tumours was very low $(\mathrm{n}=11,39.29 \%)$, which could have compromised the sensitivity of the techniques analysed in the identification of malignancy. Lastly, colour doppler and Stress elastography techniques are currently only available at research centres and university hospitals, temporarily limiting the clinical application of these exams. 


\section{CONCLUSION}

In conclusion, B-mode and Doppler ultrasonographic evaluation may assist stress elastography in accurate prediction of malignancy of canine tumour masses and inclusion of above ultrasonographic examinations in veterinary clinical oncology and research is highly recommended; since it allows fast, non-invasive, and complication free malignancy prediction of canine tumour masses.

\section{ACKNOWLEDGEMENTS}

The authors would like to thank the Hon'ble ViceChancellor, Odisha University of Agriculture and Technology; Dean, College of Veterinary Science and Animal Husbandry and Director, Teaching Veterinary Clinical Complex, Odisha University of Agriculture and Technology, Bhubaneswar, Odisha, India for providing the necessary facilities for the research.

\section{REFERENCES}

Carvalho, F., Cintra, T.C.F. and Chammas, M.C. 2015. Elastography: Principles and considerations for clinical research in veterinary medicine cibele. J. Vet. Med. Anim. Health, 7(3): 99-110.

Cassali, G.D., Lavalle, G.E., De Nardi, A.B., Ferreira, E., Bertagnolli, A.C., Estrela-Lima, A., Alessi, A.C., Daleck, C.R., Salgado, B.S., Fernandes, C.G. and Sobral, R.A. 2011. Consensus for the diagnosis, prognosis and treatment of canine mammary tumors. Braz. J. Vet. Pathol., 4(2): 153-180.

Davoudi, Y., Borhani, B., Rad, M.P. and Matin, N. 2014. The role of Doppler sonography in distinguishing malignant from benign breast lesions. J. Med. Ultrasound, 22(2): 92-95.

de Bulnes, A.G., Fernandez, P.G., Aguirre, A.M. and de la Muela, M.S. 1998. Ultrasonographic imaging of canine mammary tumours. Vet. Rec., 143(25): 687-689.

Díaz, C.D.A., Estrada, L.M., Pascual, A.M., Pelayo, A.A., and Ortega, L.M. 2018. Adenomyoepithelioma of the breast: Report of four cases and literature review. Rev. Esp. Patol., 51(1): 55-60.

Dixon, J.M., Walsh, J., Paterson, D., and Chetty, U. 1992. Colour Doppler ultrasonography studies of benign and malignant breast lesions. Br. J. Surg., 79(3): 259-260.

Feliciano, M.A.R., Silva, A.S., Peixoto, R.V.R., Galera, P.D., and Vicente, W.R.R. 2012a. Clinical, histopathological and immunohistochemical study of mammary neoplasm in bitches. Arq. Bras. Med. Vet. Zootec., 64(5): 1094-1100.
Feliciano, M.A.R., Vicente, W.R.R., and Silva, M.A.M. 2012b. Conventional and Doppler ultrasound for the differentiation of benign and malignant canine mammary tumours. J. Small Anim. Pract., 53(6): 332-337.

Feliciano, M.A.R., Uscategui, R.A.R., Maronezi, M.C., Simões, A.P.R., Silva, P., Gasser, B., Pavan, L., Carvalho, C.F., Canola, J.C. and Vicente, W.R.R. 2017. Ultrasonography methods for predicting malignancy in canine mammary tumors. PloS One, 12(5): e0178143.

Feliciano, M.A.R., Ramirez, R.A.U., Maronezi, M.C., Maciel, G.S., Avante, M.L., Senhorello, I.L.S., Mucédola, T., Gasser, B., Carvalho, C.F. and Vicente, W.R.R. 2018. Accuracy of four ultrasonography techniques in predicting histopathological classification of canine mammary carcinomas. Vet. Radiol. Ultrasound, 59(4): 444-452.

Forrest, L.J. 2007. Diagnostic imaging in oncology. In: Withrow and Macewen's small animal clinical oncology, $4^{\text {th }}$ Ed., (Eds) Withrow, S. and Vail, D. Saunders Elsevier, St. Louis, Missouri, pp. 97-111

Gokhale, S. 2009. Ultrasound characterization of breast masses. Indian J. Radiol. Imaging, 19(3): 242.

Nair, B.C., Saikumar, G., Sharma, R., and Paliwal, O.P. 2007. A study on spontaneous canine neoplasms in Bareilly, UP. Indian J. Vet. Pathol., 31(2): 166-168.

Nyman, H.T., Kristensen, A.T., Lee, M.H., Martinussen, T., and Mcevoy, F.J. 2006a. Characterization of canine superficial tumors using gray-scale B mode, color flow mapping, and spectral doppler ultrasonography - a multivariate study. Vet. Radiol. Ultrasound, 47(2): 192-198.

Nyman, H.T., Nielsen, O.L., McEvoy, F.J., Lee, M.H., Martinussen, T., Hellmén, E., and Kristensen, A.T. 2006 b. Comparison of B-mode and Doppler ultrasonographic findings with histologic features of benign and malignant mammary tumors in dogs. AM. J. Vet. Res., 67(6): 985-991.

Ohlerth, S. and Kaser-Hotz, B. 2003. A review of Doppler sonography for the assessment of tumour vascularity. Vet. Comp. Oncol.,1: 121-130.

Patel, M.P., Ghodasara, D.J., Raval, S.H., and Joshi, B.P. 2019. Incidence, Gross Morphology, Histopathology and Immunohistochemistry of Canine Mammary Tumors. Indian J. Vet. Sci. Biotechnol., 14(4): 40-44.

Snedecor, G. W. and Cochran, W. G. 2004. Statistical methods. $8^{\text {th }}$ Edn., East West Press Pvt. Ltd., New Delhi.

Soler, M., Dominguez, E., Lucas, X., Novellas, R., GomesCoelho, K.V., Espada, Y. and Agut, A. 2016. Comparison between ultrasonographic findings of benign and malignant 
canine mammary gland tumours using B-mode, colour Doppler, power Doppler and spectral Doppler. Res. Vet. Sci., 107: $141-146$.

Stuhrmann, M., Aronius, R., and Schietzel, M., 2000. Tumor vascularity of breast lesions: potentials and limits of contrastenhanced Doppler sonography. A.J.R. Am. J. Roengenol., 175(6): 1585-1589.
Vascellari, M., Baioni, E., Ru, G., Carminato, A. and Mutinelli, F. 2009. Animal tumour registry of two provinces in northern Italy: incidence of spontaneous tumours in dogs and cats. B.M.C. Vet. Res., 5(1): 39 . 
